\title{
FRANÇOISE VINCENT
}

\section{Une note sur les fonctions invariantes}

Annales de la faculté des sciences de Toulouse $6^{e}$ série, tome $6, \mathrm{n}^{\circ} 2$ (1997), p. 357-363

<http://www.numdam.org/item?id=AFST_1997_6_6_2_357_0>

(C) Université Paul Sabatier, 1997, tous droits réservés.

L'accès aux archives de la revue «Annales de la faculté des sciences de Toulouse » (http://picard.ups-tlse.fr/ annales/) implique l'accord avec les conditions générales d'utilisation (http://www.numdam.org/conditions). Toute utilisation commerciale ou impression systématique est constitutive d'une infraction pénale. Toute copie ou impression de ce fichier doit contenir la présente mention de copyright.

\section{Numdam}

Article numérisé dans le cadre du programme Numérisation de documents anciens mathématiques http://www.numdam.org/ 


\title{
Une note sur les fonctions invariantes ${ }^{(*)}$
}

\author{
FRANÇOISE VINCENT ${ }^{(1)}$
}

\begin{abstract}
RÉSUMÉ. - Soit $\mathfrak{g}=\mathfrak{k}+\mathfrak{p}$ une décomposition de Cartan d'une algèbre de Lie semi-simple réelle $\mathfrak{g}, \mathfrak{a}$ un sous-espace de Cartan de $\mathfrak{p}$ et $W$ le groupe de Weyl associé à la paire $(\mathfrak{g}, \mathfrak{a})$. $G$ désignera un groupe de Lie connexe d'algèbre de Lie $g$ et $K$, le sous-groupe de Lie connexe de $G$ d'algèbre de Lie $\mathfrak{k}$. On montre que l'opération de restriction à $\mathfrak{a}$ des fonctions réelles définies dans $\mathfrak{p}$ est une bijection de l'ensemble des fonctions $f: \mathfrak{p} \rightarrow \mathbb{R}$ convexes et $K$-invariantes sur celui des fonctions $f_{0}: \mathfrak{a} \rightarrow \mathbb{R}$ convexes et $W$-invariantes. Ceci généralise de récents résultats de $\mathrm{B}$. Dacorogna et $\mathrm{H}$. Koshigoe sur les fonctions convexes invariantes par rotation.
\end{abstract}

Abstract. - Let $\mathfrak{g}=\mathfrak{k}+\mathfrak{p}$ a Cartan decomposition of a real semisimple Lie algebra $\mathfrak{g}, \mathfrak{a}$ a Cartan subspace, $W$ the Weyl group associated with the pair $(\mathfrak{g}, \mathfrak{a})$. $G$ will describe a connex Lie group with Lie algebra $\mathfrak{g}$ and $K$, the connex Lie subgroup of $G$, with Lie algebra $k$. We show that the operation of restriction to $\mathfrak{a}$ of the real functions defined in $\mathfrak{p}$, is a one to one mapping of the set of functions $f: \mathfrak{p} \rightarrow \mathbb{R}$ convex and $K$ invariant onto this of the functions $f_{0}: \mathfrak{a} \rightarrow \mathbb{R}$ convex and $W$-invariant. This generalises B. Dacorogna and H. Koshigoe's recent results on the convex rotationally invariant functions.

\section{Introduction}

Cette note propose une généralisation de résultats démontrés par B. Dacorogna et H. Koshigoe dans [1], concernant les fonctions convexes invariantes, particulièrement du théorème 1.1 de la référence citée, dont on rappelle l'énoncé avec les notations suivantes : $\mathcal{M}_{n}$ est l'espace vectoriel des matrices $n \times n$ à coefficients réels et $\mathrm{SO}(n)$ est le groupe des matrices orthogonales $n \times n$, de déterminant égal à 1 .

(*) Reçu le 26 juin 1995

(1) Département de Mathématiques, Université de La Rochelle, F-17000 La Rochelle. URA CNRS, Groupes et Géométrie de l'Université de Poitiers e-mail : fvincent@iniv-lr.fr 
THÉORÈME 1.1 [1]. - Soit $f$ une application de $\mathcal{M}_{2}$ dans $\mathbb{R}$ satisfaisant l'hypothèse suivante :

$$
f(U \xi V)=f(\xi) \text { pour tous } \xi \in \mathcal{M}_{2}, U, V \in \mathrm{SO}(2) .
$$

Alors, les conditions suivantes sont équivalentes :

(i) $f$ est convexe;

(ii) la restriction de $f$ au sous-espace vectoriel de $\mathcal{M}_{2}$ constitué des matrices diagonales est convexe.

Les auteurs affirment que la plupart de leurs résultats s'étendent au cas des matrices $n \times n$ avec $n \geq 2$ et qu'ils se restreignent au cas $n=2$ dans un but de simplicité.

Soit, plus généralement, $\mathfrak{g}=\mathfrak{k}+\mathfrak{p}$ une décomposition de Cartan d'une algèbre de Lie semi-simple réelle $\mathfrak{g}$. On désigne par $G$ un groupe de Lie connexe d'algèbre de Lie $\mathfrak{g}$ et par $K$ le sous-groupe de Lie connexe de $G$, d'algèbre de Lie $\mathfrak{k}$. Le groupe $K$ (qui est en fait un sous-groupe compact de $G$ ) opère naturellement (linéairement et continûment) dans l'espace vectoriel $\mathfrak{p}$, au moyen de la représentation adjointe que l'on notera Ad. Cette représentation linéaire de $K$ dans $\mathfrak{p}$ est souvent appelée la représentation tangente de l'espace riemannien symétrique $G / K$. Soit $\mathfrak{a}$ un sous-espace de Cartan de $\mathfrak{p}$ (c'est un sous-espace de $\mathfrak{p}$ qui est transverse aux orbites de dimension maximale de $K$ dans $\mathfrak{p}$ ) et soit $W$ le groupe de Weyl de la paire $(\mathfrak{g}, \mathfrak{a})$, considéré comme un sous-groupe du groupe linéaire $\mathrm{GL}(\mathfrak{a})$ de l'espace vectoriel $\mathfrak{a}(W$ est en fait un sous-groupe fini de $\mathrm{GL}(\mathfrak{a})$ qui est engendré par les réflexions). On note $\mathcal{R}_{\mathfrak{a}}$ l'opération de restriction à a des fonctions réelles définies dans $\mathfrak{p}$. On a alors :

THÉORÈME 1.2. $-\mathcal{R}_{\mathfrak{a}}$ induit une bijection de l'ensemble des fonctions $f: \mathfrak{p} \rightarrow \mathbb{R}$ qui sont convexes et $K$-invariantes sur celui des fonctions $f_{0}: \mathfrak{a} \rightarrow \mathbb{R}$ qui sont convexes et $W$-invariantes. En particulier, si $f: \mathfrak{p} \rightarrow \mathbb{R}$ est $K$-invariante, alors $f$ est convexe si et seulement si sa restriction $\grave{a} \mathfrak{a}$ est convexe.

Exemple 1.- On prend $G=\mathrm{SL}(n)$ (groupe des matrices $n \times n$ de déterminant 1 , à coefficients réels). Dans cette situation, $K$ s'identifie à $\mathrm{SO}(n), \mathfrak{p}$ s'identifie à l'espace vectoriel des matrices réelles symétriques de trace nulle, l'opération de $K$ dans $\mathfrak{p}$ est la suivante :

$$
\operatorname{Ad}(x)(X)=x X x^{-1} \quad \text { pour tous } X \in \mathfrak{p}, x \in \mathrm{SO}(n) .
$$


On peut prendre pour sous-espace de Cartan $\mathfrak{a}$, le sous-espace de $\mathfrak{p}$ constitué des matrices diagonales.

Exemple 2.- Soit $G$ la composante neutre (composante connexe de l'unité) du groupe orthogonal de la forme quadratique (sur $\mathbb{R}^{n}$ ) :

$Q\left(x_{1}, \ldots, x_{n}\right)=x_{1}^{2}+\cdots+x_{p}^{2}-x_{p+1}^{2}-\cdots-x_{p+q}^{2}, \quad 0<p \leq q$ et $p+q=n$.

Ici $K$ s'identifie au groupe produit $\mathrm{SO}(p) \times \mathrm{SO}(q), \mathfrak{p}$ s'identifie à l'espace vectoriel $\mathcal{M}_{p, q}$ des matrices $p \times q$, à coefficients réels, et l'opération de $K$ dans $\mathfrak{p}$ est la suivante :

$$
\operatorname{Ad}(x, y)(X)=x X y^{-1} \quad \text { pour tous }(x, y) \in \mathrm{SO}(p) \times \mathrm{SO}(q) \text { et } X \in \mathcal{M}_{p, q} \text {. }
$$

On peut prendre pour sous-espace de Cartan a, le sous-espace de $\mathcal{M}_{p, q}$ constitué par les matrices de la forme :

$$
X=\left(\begin{array}{ccccccc}
a_{1} & 0 & \ldots & 0 & 0 & \ldots & 0 \\
0 & a_{2} & \ldots & 0 & \vdots & \ldots & 0 \\
\vdots & \vdots & \ddots & \vdots & \vdots & & \vdots \\
0 & 0 & \ldots & a_{p} & 0 & \ldots & 0
\end{array}\right)
$$

$a_{i} \in \mathbb{R}, 1 \leq i \leq p$. Lorsque $p=q=2$, la dernière partie du théorème 1.2 est exactement le théorème 1.1 de Dacorogna et Koshigoe. On notera que dans ce cas, aprés identification de l'espace des matrices diagonales avec $\mathbb{R}^{2}$, le groupe de Weyl $W$ est le groupe (à 4 éléments) engendré par les réflexions orthogonales par rapport aux deux bissectrices des axes de coordonnées et que ces transformations linéaires de $\mathbb{R}^{2}$ apparaissent explicitement dans les démonstrations de [1]. Toutefois la démonstration que l'on donne du théorème $1.2 \mathrm{n}$ 'est pas de même nature : elle découle presque immédiatement d'un théorème de convexité de Kostant [3, theorem 8.2 ] que l'on présente dans la section suivante.

On renvoit à [2] pour les notions de base concernant les espaces riemanniens symétriques irréductibles, chacun d'eux fournit un exemple où s'applique le théorème 1.2 . 


\section{Le théorème de convexité de Kostant}

On reprend la décomposition $\mathfrak{g}=\mathfrak{k}+\mathfrak{p}$ de l'introduction. La restriction $\grave{a} \mathfrak{p}$ de la forme bilinéaire de Killing définit sur $\mathfrak{p}$ un produit scalaire qui est $K$-invariant. Les notions d'orthogonalité utilisées plus loin seront relatives à ce produit scalaire.

- Soit $X$ un élément régulier de $\mathfrak{p}$ dont la $K$-orbite $K \cdot X$ est de dimension maximale, et soit $T_{X}$ l'espace tangent en $X$ à cette orbite. L'orthogonal $T_{X}^{\frac{1}{X}}$ de $T_{X}$ est un sous-espace de Cartan, noté $\mathfrak{a}$.

- Le sous-espace de Cartan a étant choisi, on considère le sous-groupe $M$ de $K$ constitué par les éléments $x$ de $K$ tels que $\operatorname{Ad}(x)(\mathfrak{a})=\mathfrak{a}$ (c'est le normalisateur de $\mathfrak{a}$ dans $K$ ). Par sa définition, le groupe $M$ opère linéairement dans l'espace vectoriel a. On désigne par $W$ l'image de $M$ dans $\mathrm{GL}(\mathfrak{a})$ qui résulte de cette opération de $M$ dans $\mathfrak{a}$. C'est le groupe de Weyl de $(\mathfrak{g}, \mathfrak{a})$.

On sait alors (voir [2]) que :

i) toute orbite $\Omega$ de $K$ dans $\mathfrak{p}$ rencontre $\mathfrak{a}: \Omega \cap \mathfrak{a} \neq \emptyset$;

ii) Si $\Omega$ est une orbite de $K$ dans $\mathfrak{p}$, alors $\Omega \cap \mathfrak{a}$ est une orbite de $W$ dans $\mathfrak{a}$.

On peut maintenant énoncer le théorème de Kostant.

THÉORÈme 2.1 [3]. - Soit $\pi$ l'opérateur de projection orthogonale de $\mathfrak{p}$ sur $\mathfrak{a}$. Soit $\Omega$ une orbite de $K$ dans $\mathfrak{p}$. Alors la projection orthogonale $\pi(\Omega)$ de $\Omega$ dans a est l'enveloppe convexe de $\Omega \cap \mathfrak{a}$.

On en déduit le résultat suivant.

Lemme 2.1.- Soit $f: \mathfrak{p} \rightarrow \mathbb{R}$ une fonction $K$-invariante. On suppose que sa restriction $f_{0} \grave{a} \mathfrak{a}$ est convexe. Alors, pour tout $X$ dans $\mathfrak{p}$, pour tout $Y$ dans $\pi(K \cdot X)$, on $a$ :

$$
f_{0}(Y) \leq f(X) .
$$


Démonstration. - Soient $X$ dans $\mathfrak{p}$ et $Y$ dans $\pi(K \cdot X)$. D'après le théorème de Kostant, il existe un nombre fini $Y_{1}, \ldots, Y_{m}$ d'éléments de $K \cdot X \cap \mathfrak{a}$ et des réels positifs $\lambda_{1}, \ldots, \lambda_{m}$, de somme 1 , tels que

$$
Y=\sum_{i=1}^{m} \lambda_{i} Y_{i} .
$$

La fonction $f_{0}$ étant convexe, il vient :

$$
f_{0}(Y) \leq \sum_{i} \lambda_{i} f_{0}\left(Y_{i}\right)
$$

mais $Y_{i} \in K \cdot X$ et $f$ est $K$-invariante, donc $f_{0}\left(Y_{i}\right)=f(X)$ et $f_{0}(Y) \leq f(X)$.

\section{Démonstration du théorème 1.2}

- Soit $f_{0}: \mathfrak{a} \rightarrow \mathbb{R}$ une fonction $W$-invariante. Alors $f_{0}$ se prolonge de manière unique, en une fonction $K$-invariante $f: \mathfrak{p} \rightarrow \mathbb{R}$. On remarque en effet que si $X$ est un élément de $\mathfrak{p}, K \cdot X \cap \mathfrak{a}$ est une $W$-orbite, de sorte que $f_{0}$ prend une valeur constante sur $K \cdot X \cap \mathfrak{a}$, et que, si on pose $f(X)=f_{0}(K \cdot X \cap \mathfrak{a})$, on définit bien une fonction $f: \mathfrak{p} \rightarrow \mathbb{R}$. Il est immédiat que $f$ est $K$-invariante : si $Y=\operatorname{Ad}(x)(X)$ avec $x \in K$, on a $K \cdot X=K \cdot Y$, d'où $K \cdot X \cap \mathfrak{a}=K \cdot Y \cap \mathfrak{a}$ et $f(X)=f(Y)$. Il est immédiat que $f\rceil_{\mathfrak{a}}=f_{0}$ : si $X \in \mathfrak{a}, K \cdot X \cap \mathfrak{a}$ étant une $W$-orbite, ne peut être que $W \cdot X$, d'où $f(X)=f_{0}(W \cdot X)=f_{0}(X)$. Enfin, le prolongement $K$-invariant de $f_{0}$ est unique (ceci résulte du fait que toutes les $K$-orbites rencontrent $\mathfrak{a}$ ).

- Soit $f_{0}: \mathfrak{a} \rightarrow \mathbb{R}$ une fonction $W$-invariante et convexe. Soit $f: \mathfrak{p} \rightarrow \mathbb{R}$ le prolongement $K$-invariant de $f_{0}$, construit ci-dessus. Montrons que $f$ est convexe. Soient $X_{1}$ et $X_{2}$ dans $\mathfrak{p}$ et $t$ dans $[0,1]$. La $K$-orbite de $t X_{1}+(1-t) X_{2}$ rencontre a. Il existe donc $x$ dans $K$ tel que $Z=$ $\operatorname{Ad}(x)\left(t X_{1}+(1-t) X_{2}\right)$ soit dans a. La fonction $f$ étant $K$-invariante, on a :

$$
f\left(t X_{1}+(1-t) X_{2}\right)=f(Z) \quad \text { et } \quad f(Z)=f_{0}(Z)
$$

car $Z \in \mathfrak{a}$. Puisque $Z \in \mathfrak{a}$,

$$
Z=\pi(Z)=t \pi\left(\operatorname{Ad}(x)\left(X_{1}\right)\right)+(1-t) \pi\left(\operatorname{Ad}(x)\left(X_{2}\right)\right),
$$


et par suite de la convexité de $f_{0}$ :

$$
f_{0}(Z) \leq t f_{0}\left(\pi\left(\operatorname{Ad}(x)\left(X_{1}\right)\right)\right)+(1-t) f_{0}\left(\pi\left(\operatorname{Ad}(x)\left(X_{2}\right)\right)\right) .
$$

D'après le lemme 2.1 :

$$
f_{0}\left(\pi\left(\operatorname{Ad}(x)\left(X_{1}\right)\right)\right) \leq f\left(X_{1}\right) \quad \text { et } \quad f_{0}\left(\pi\left(\operatorname{Ad}(x)\left(X_{2}\right)\right)\right) \leq f\left(X_{2}\right),
$$

on a bien $f(Z) \leq t f\left(X_{1}\right)+(1-t) f\left(X_{2}\right)$ ce qui prouve la convexité de $f$.

Ainsi, toute fonction $f_{0}: \mathfrak{a} \rightarrow \mathbb{R}$, convexe et $W$-invariante se prolonge, de manière unique, en une fonction $f: \mathfrak{p} \rightarrow \mathbb{R}$, convexe et $K$-invariante.

- Dans l'autre sens, si $f: \mathfrak{p} \rightarrow \mathbb{R}$ est une fonction $K$-invariante et convexe, il est immédiat que sa restriction $f_{0}$ à a est $W$-invariante et convexe. Ceci termine la démonstration du théorème 1.2.

Remarque 3.1. - Il est immédiat que si $f$ et $g$ sont deux fonctions réelles $K$-invariantes sur $\mathfrak{p}, f_{0}$ et $g_{0}$ leurs restrictions respectives à $\mathfrak{a}$, on a $: f \leq g$ si et seulement si $f_{0} \leq g_{0}$. Autrement dit, la bijection induite par $\mathcal{R}_{\mathfrak{a}}$ entre l'ensemble des fonctions convexes et $K$-invariantes sur $\mathfrak{p}$ d'une part et celui des fonctions convexes et $W$-invariantes sur a d'autre part est un isomorphisme d'ensembles ordonnés. Ceci semble avoir des conséquences intéressantes dans la pratique pour la détermination de l'enveloppe convexe $\mathcal{C} f$ d'une fonction $K$-invariante $f$ (on rappelle que $\mathcal{C} f$ est l'enveloppe supérieure de l'ensemble des fonctions convexes majorées par $f$, (voir [1, sect. 4]). Le lemme suivant généralise la partie $A$ du théorème 4.2 de [1].

LeMme 3.1.- Soit $f: \mathfrak{p} \rightarrow \mathbb{R}$ une fonction $K$-invariante. Alors $\mathcal{R}_{\mathfrak{a}}(\mathcal{C} f)=\mathcal{C}\left(\mathcal{R}_{\mathfrak{a}} f\right)$ ou encore $\left.\left.\mathcal{C} f\right\rceil_{\mathfrak{a}}=\mathcal{C}(f\rceil_{\mathfrak{a}}\right)$.

Démonstration. - Posons $f_{0}=f \uparrow_{\mathfrak{a}}$ et $g_{0}=\mathcal{C} f_{0}$. La fonction $f$ étant $K$-invariante, il vient que $\mathcal{C} f$ est elle-même $K$-invariante. De même $g_{0}$ est $W$-invariante. Il revient donc au même de montrer que $\mathcal{C} f$ coïncide avec l'unique prolongement $K$-invariant $g$ de $g_{0}$. Pour cela, on remarque d'abord : $\mathcal{C} f$ est convexe et $\mathcal{C} f \leq f ; \operatorname{donc} \mathcal{C} f\rceil_{\mathfrak{a}} \leq f_{0}$, ainsi $\left.\mathcal{C} f\right\rceil_{\mathfrak{a}} \leq \mathcal{C} f_{0}=g_{0}$ et $\mathcal{C} f \leq g$. On remarque ensuite : $g_{0}=\mathcal{C} f_{0}$ est convexe et $g_{0} \leq f_{0}$, donc $g$ est convexe et $g \leq f$, ainsi $g \leq \mathcal{C} f$. On a bien $g=\mathcal{C} f$.

La signification du lemme est la suivante : pour trouver l'enveloppe $\mathcal{C} f$ d'une fonction $K$-invariante, il suffit de restreindre $f$ en $f_{0}$ sur a, de trouver 
l'enveloppe $\mathcal{C} f_{0}$ et enfin de prolonger $\mathcal{C} f_{0}$ en une fonction $K$-invariante sur $\mathfrak{p}$. L'intérêt réside en ce que la dimension de a peut être considérablement plus petite que celle de $\mathfrak{p}$. On signale que la dimension de $\mathfrak{a}$ est ce qu'on appelle le rang de l'espace symétrique $G / K$ et qu'elle est connue pour chacun des espaces symétriques de la classification figurant dans [2].

\section{Références}

[1] Dacorogna (B.) et Koshigoe (H.) .- On the different notions of convexity for rotationally invariant functions, Ann. de la Faculté des Sciences de Toulouse, II, $\mathrm{n}^{\circ} 2$ (1993), pp. 163-184.

[2] HeLgason (S.) .- Differential geometry, Lie groups, and symmetric spaces, Academic Press, 1978.

[3] Kostant (B.) .- On convexity, the Weyl group and the Iwasawa decomposition, Ann. Sci. École Normale Sup. 6 (1973), pp. 413-455. 